University of South Carolina

Scholar Commons

Spring 2-2014

\title{
Modulation of Leukocyte Infiltration and Phenotype in Microporous Tissue Engineering Scaffolds via Vector Induced II-10 Expression
}

\author{
R. Michael Gower \\ University of South Carolina - Columbia, gowerrm@mailbox.sc.edu \\ Ryan M. Boehler \\ Samira M. Azarin \\ Christine F. Ricci \\ Joshua N. Leonard
}

See next page for additional authors

Follow this and additional works at: https://scholarcommons.sc.edu/eche_facpub

Part of the Biomedical Engineering and Bioengineering Commons, and the Chemical Engineering Commons

Publication Info

Published in Biomaterials, Volume 35, Issue 6, Spring 2014, pages 2024-2031.

(c) Biomaterials, 2014, Elsevier.

Gower, R., Boehler, R., Azarin, S., Ricci, C., Leonard, J., \& Shea, L. (2014). Modulation of leukocyte infiltration and phenotype in microporous tissue engineering scaffolds via vector induced IL-10 expression. Biomaterials, 35(6), 2024-2031.

doi: 10.1016/j.biomaterials.2013.11.036

This Article is brought to you by the Chemical Engineering, Department of at Scholar Commons. It has been accepted for inclusion in Faculty Publications by an authorized administrator of Scholar Commons. For more information, please contact digres@mailbox.sc.edu. 


\section{Author(s)}

R. Michael Gower, Ryan M. Boehler, Samira M. Azarin, Christine F. Ricci, Joshua N. Leonard, and Lonnie D. Shea 
University of South Carolina

Scholar Commons

Spring 2-2014

Modulation of leukocyte infiltration and phenotype in microporous tissue engineering scaffolds via vector induced IL-10 expression

Michael Gower

Follow this and additional works at: https://scholarcommons.sc.edu/acc_facpub

Part of the Biomedical Engineering and Bioengineering Commons, and the Chemical Engineering Commons 


\title{
Modulation of leukocyte infiltration and phenotype in microporous tissue engineering scaffolds via vector induced IL-10 expression
}

\author{
R. Michael Gower ${ }^{a}$, Ryan M. Boehler ${ }^{a}$, Samira M. Azarin ${ }^{a}$, Christine F. Ricci ${ }^{a}$, \\ Joshua N. Leonard ${ }^{\mathrm{a}, \mathrm{d}, \mathrm{e}}$, Lonnie D. Shea ${ }^{\mathrm{a}, \mathrm{b}, \mathrm{c}, \mathrm{d}, \mathrm{e}, *}$ \\ ${ }^{a}$ Department of Chemical and Biological Engineering, McCormick School of Engineering, Northwestern University, Evanston, IL, USA \\ ${ }^{\mathrm{b}}$ Department of Biomedical Engineering, McCormick School of Engineering, Northwestern University, Evanston, IL, USA \\ ${ }^{c}$ Institute for BioNanotechnology in Medicine (IBNAM), Northwestern University, Chicago, IL, USA \\ ${ }^{\mathrm{d}}$ Robert H. Lurie Comprehensive Cancer Center, Northwestern University, Chicago, IL, USA \\ ${ }^{\mathrm{e}}$ Chemistry of Life Processes Institute (CLP), Northwestern University, Evanston, IL, USA
}

\section{A R T I C L E I N F O}

\section{Article history:}

Received 19 October 2013

Accepted 13 November 2013

Available online 3 December 2013

\section{Keywords:}

Inflammation

Immunomodulation

Gene therapy

Flow cytometry

Scaffold

Leukocyte

\begin{abstract}
A B S T R A C T
Biomaterial scaffolds are central to many tissue engineering strategies as they create a space for tissue growth and provide a support for cell adhesion and migration. However, biomaterial implantation results in unavoidable injury resulting in an inflammatory response, which can impair integration with the host and tissue regeneration. Toward the goal of reducing inflammation, we investigated the hypothesis that a lentiviral gene therapy-based approach to localized and sustained IL-10 expression at a scaffold could modulate the number, relative proportions, and cytokine production of infiltrating leukocyte populations. Flow cytometry was used to quantify infiltration of six leukocyte populations for 21 days following implantation of PLG scaffolds into intraperitoneal fat. Leukocytes with innate immune functions (i.e., macrophages, dendritic cells, neutrophils) were most prevalent at early time points, while T lymphocytes became prevalent by day 14 . Reporter gene delivery indicated that transgene expression persisted at the scaffold for up to 28 days and macrophages were the most common leukocyte transduced, while transduced dendritic cells expressed the greatest levels of transgene. IL-10 delivery decreased leukocyte infiltration by $50 \%$ relative to controls, increased macrophage IL-10 expression, and decreased macrophage, dendritic cell, and CD4 T cell IFN- $\gamma$ expression. Thus, IL-10 gene delivery significantly decreased inflammation following scaffold implant into the intraperitoneal fat, in part by modulating cytokine expression of infiltrating leukocytes.
\end{abstract}

(C) 2013 Elsevier Ltd. All rights reserved.

\section{Introduction}

Biomaterial scaffolds are central to many tissue-engineering strategies and are employed to create an environment to promote regeneration. Scaffolds create a space for tissue growth, support host cell infiltration, and promote vascularization, all of which are necessary for regeneration of lost or injured tissues. Scaffolds also function as a platform for cell transplantation, such as islets for diabetes therapies, retaining the transplanted cells within a defined anatomical location, and as a support for cell infiltration that integrates transplanted cells with the host. Furthermore, scaffolds may be modified with biological signals, such as extracellular matrix (ECM) proteins to modulate cell adhesion and migration, or inductive factors to stimulate or direct tissue growth. The biological

\footnotetext{
* Corresponding author. Department of Chemical and Biological Engineering, Northwestern University, 2145 Sheridan Rd./Tech E136, Evanston, IL 60208-3120, USA. Tel.: +1 847491 7043; fax: +1 8474913728

E-mail address: 1-shea@northwestern.edu (L.D. Shea).
}

signals presented within the scaffold are ultimately aimed at creating an environment for which endogenous or transplanted progenitor cells can promote regeneration.

The innate immune response to implanted biomaterials plays a crucial role in the post-injury microenvironment that can determine the extent of regeneration [1,2]. This immune response is initiated by tissue resident leukocytes, including macrophages and dendritic cells, which respond to the foreign material by secreting chemokines that recruit peripheral leukocytes, initially neutrophils and monocytes, to the implant site [3-5]. Infiltrating leukocytes become activated at the implant, leading to release of proinflammatory cytokines, such as IL- $1 \beta, \mathrm{TNF}-\alpha$, and IFN- $\gamma[6,7]$, which are well known to inhibit tissue regeneration or limit the survival of transplanted tissue [8,9]. Conversely, up-regulation of anti-inflammatory cytokines such as IL-10 is associated with resolution of inflammation [10] and survival of transplanted cells [11]. Thus, modulating the inflammatory response has the potential to enhance regenerative therapies. 
Localized delivery of immunomodulatory factors has emerged as a strategy for controlling the immune response. Cells and tissues have been engineered ex vivo to express immunomodulatory factors prior to transplantation, a strategy that has decreased rejection of cell and organ transplants [12-14]. In addition, tissueengineering scaffolds have been designed to release proteins to enhance angiogenesis or modulate inflammatory cell responses $[15,16]$. However, a major hurdle for this approach is protein stability in the delivery system. More recently, gene delivery from biomaterial scaffolds has been demonstrated as a versatile approach to target infiltrating cells as bioreactors for the localized production of factors [17]. Furthermore, gene delivery from biomaterials has been shown to transduce leukocytes, providing the opportunity to directly modulate the innate response [18-20]. For example, plasmid-mediated production of IL-10 has been shown to decrease the inflammatory response to stem cells in collagen scaffolds thus increasing stem cell survival [11,21].

In this report, we investigated the hypothesis that a lentiviral gene therapy-based approach to localized and sustained IL-10 expression could modulate the number, relative proportions, and cytokine production of leukocyte populations infiltrating poly(lactide-co-glycolide) (PLG) scaffolds. Using flow cytometry, we quantified the infiltration of six major leukocyte populations into PLG scaffolds implanted into the intraperitoneal (IP) fat. Bioluminescence imaging was employed to characterize the level and duration of transgene expression within PLG scaffolds implanted with luciferase viruses, while flow cytometry was used to identify the leukocyte populations expressing the transgene in scaffolds delivering tdTomato viruses. Finally, flow cytometry was employed to characterize leukocyte populations and their cytokine expression within PLG scaffolds following viral IL-10 delivery. Development of strategies to modulate the inflammatory response has the potential to create a more permissive environment that can enhance numerous applications within regenerative medicine.

\section{Materials and methods}

2.1. Animals

Animal studies were performed in accordance with the NIH Guide for the Care and Use of Laboratory Animals, and protocols were approved by the IACUC at Northwestern University. Male CD1 mice weighing 19-24 g were acquired from Charles River and maintained in conventional housing.

\subsection{Virus production}

DNA encoding for murine IL-10 (Openbiosystems) or Luciferase (Promega) were cloned into a self-inactivating lentiviral vector [22]. Lentivirus was produced in HEK293T cells grown in DMEM with $10 \% \mathrm{FBS}$ at $37{ }^{\circ} \mathrm{C}$, and $5 \% \mathrm{CO}_{2}$. The lentiviral packaging vectors (pMDL-GagPol, pRSV-Rev, pIVS-VSV-G) were co-transfected with the lentiviral vector into 293T cells using Lipofectamine 2000 (Life Technologies). After $48 \mathrm{~h}$ of transfection, the supernatant was collected and filtered ( $0.45 \mathrm{micron})$. Viruses were then concentrated using PEG-it (System Biosciences) and suspended using sterile PBS. The virus titer was determined using a qPCR Lentivirus Titer Kit (Applied Biological Materials). Typical titers ranged between $2 \times 10^{9}$ and $8 \times 10^{9}$ particles $/ \mathrm{mL}$.

\subsection{Scaffold fabrication}

Poly(lactide-co-glycolide) (PLG) microspheres were prepared as previously described $[23,24]$. Briefly, PLG (75:25 mol ratio D,L-lactide to glycolide, $0.76 \mathrm{dL} / \mathrm{g}$ ) (Lakeshore Biomaterials) was dissolved in dichloromethane to make a $6 \%(\mathrm{w} / \mathrm{w})$ solution, which was then emulsified in $1 \%$ poly(vinyl alcohol) to create microspheres. The microspheres were collected by centrifugation, washed with deionized water, and lyophilized overnight. Microspheres were combined in a 1:30 ratio with $\mathrm{NaCl}$ particles $250-425 \mu \mathrm{m}$ diameter. The mixture was pressed in a steel die at 1500 pounds per square inch (psi) and gas-foamed after equilibration to $800 \mathrm{psi}$ under $\mathrm{CO}_{2}$ gas. Salt particles were removed by immersion in deionized water with repeated washing. Scaffolds were sterilized in $70 \%$ ethanol and then washed twice in sterile phosphate buffered saline (PBS; Life Technologies). $2 \times 10^{7}$ viral particles (in PBS 2-10 $\mu \mathrm{L}$ ) were added to the scaffold using a micropipette. Scaffolds were immediately frozen on dry ice and stored in $-80^{\circ} \mathrm{C}$ freezer until implantation.

\subsection{In vitro cell studies}

Scaffolds loaded with virus were seeded with murine RAW 264.7 macrophages (ATCC) and incubated for $4 \mathrm{~h}$ at $37{ }^{\circ} \mathrm{C}$. Scaffolds were then transferred into wells containing fresh media and imaged daily. Luciferase expression was measured by adding d-luciferin ( $1 \mathrm{~mm}$; Caliper) to the well $4 \mathrm{~h}$ before imaging on the IVIS Spectrum (Caliper). The signal intensity was reported as an integrated light flux (photons/s) as determined by Living Image Software (Caliper).

For cytokine production assays, scaffolds loaded with virus were seeded with RAW 264.7 macrophages and cultured for 3 days prior to the addition of lipopolysaccharide (100 ng/ml; Sigma). Twenty-four hours after addition of lipopolysaccharide, supernatants were collected. TNF- $\alpha$ and IL-10 production was measured by ELISA (R\&D systems).

\subsection{Scaffold implantation}

Mice received bilateral scaffold implants in the IP fat as previously described [25]. Briefly, mice were anesthetized with an IP injection of ketamine $(10 \mathrm{mg} / \mathrm{kg})$ and xylazine ( $5 \mathrm{mg} / \mathrm{kg}$ ), and the abdominal midline was shaved and prepped in a sterile fashion. A midline lower abdominal incision was made, and the intraperitoneal (IP) fat pads were identified. Scaffolds were wrapped in the right and left IP fat pads, which were then inserted back into the IP cavity. Following scaffold implant, the abdominal wall was closed with a running stitch and the skin was closed with wound clips.

\subsection{Flow cytometry}

The following antibodies were purchased from Biolegend: anti-CD45 clone 30F11; anti-CD8a clone 53-6.7; anti-Gr1 clone RB6-8C5; anti-F4/80 clone BM8; anti-IL10 clone JES5-16E3; anti-TNF- $\alpha$ clone MP6-XT22; and anti-IFN- $\gamma$ clone XMG1.2. The following antibodies were purchased from eBioscience: anti-CD11b clone M1/70; anti-CD11c clone N418; and anti-IL-1 $\beta$ clone NJTEN3.

Following euthanization, scaffolds were harvested and immediately washed in ice cold Hanks Balanced Salt Solution (HBSS; Life Technologies). Scaffolds were minced and incubated in collagenase (Roche) at $37^{\circ} \mathrm{C}$ for $20 \mathrm{~min}$. The solution was then passed through a $70 \mu \mathrm{m}$ filter, washed in HBSS, and suspended in antibodies against extracellular antigens. After extracellular antibody incubation, cells were washed to remove unbound antibody. In experiments where intracellular antigens were detected, cells were then incubated with fixation buffer (eBioscience) and permeabilization buffer (eBioscience) as per the manufacturer's instructions. Cells were then incubated with antibodies to intracellular antigens. After intracellular antibody incubation, cells were washed to remove unbound antibody and analyzed on an LSRFortessa flow cytometer (BD Biosciences; San Jose, CA, USA). The entire sample from each scaffold was collected. Data was analyzed in FlowJo software (Treestar). Isotype controls were used to set gates for immunophenotyping and cells isolated from luciferase virus loaded scaffolds were used to set the gate for cells positive for tdTomato expression.

The gating scheme for flow cytometry data is depicted in Sup. Fig. 1. Cellular events were gated using forward scatter and side scatter (Sup. Fig. 1A). Leukocytes were then identified by CD45 expression (Sup. Fig. 1B). Non-viable cells were then excluded using LIVE/DEAD viability dye (Life Technologies) (Sup. Fig. 1C). CD4 positive cells (Q1) and CD8 positive cells (Q3) were then identified based on isotype controls (Sup. Fig. 1F). These cells are referred to as CD4 T cells and CD8 T cells, respectively. Cells negative for both $\mathrm{CD} 4$ and $\mathrm{CD} 8$ were then gated using $\mathrm{Gr} 1$ and F4/ 80 (Sup. Fig. 1E). Cells positive for Gr1 (Q1) are referred to as neutrophils and F4/80 positive cells (Q2 and Q3) are referred to as macrophages. Cells negative for both Gr1 and F4/80 were then gated using CD11b and CD11c (Sup. Fig. 1D). Cells positive for CD11c (Q2 and Q3) are referred to as dendritic cells. CD11b cells (Q1) are referred to explicitly in the text.

\subsection{In vivo bioluminescence imaging}

Initial studies employed a luciferase virus to monitor transgene expression over time. In vivo luciferase expression was monitored using an IVIS imaging system (Caliper, Alameda, CA). Mice were anesthetized with isoflurane and then administered an IP injection of d-luciferin (Caliper) at a dose $150 \mathrm{mg} / \mathrm{kg}$. The animals were then placed in the imaging chamber, and bioluminescence images were acquired (every $2 \mathrm{~min}$ for a total of $20 \mathrm{~min}$ ) until the peak light emission was confirmed. Brightfield images and bioluminescence images were superimposed using the Living Image software (Caliper). The signal intensity was reported as an integrated light flux (photons/s) as determined by Living Image Software.

\subsection{Statistics}

Multiple groups were compared using one-way ANOVA with the Tukey post-hoc test or by the Friedman test followed by Dunn's Multiple Comparison Test. All other comparisons were made with an unpaired $t$ test. The specific test used is specified in each figure legend. All analysis was carried out using GraphPad Prism. 
A

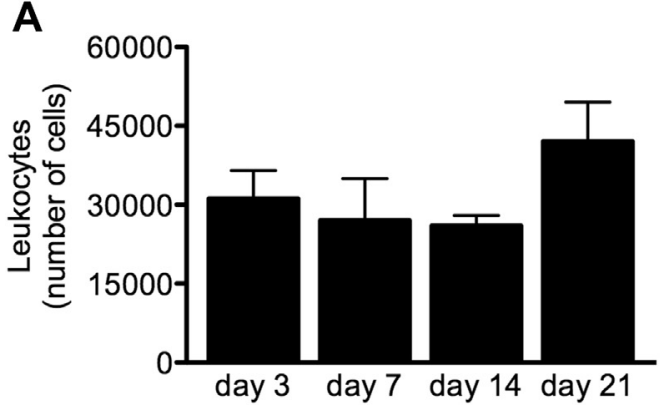

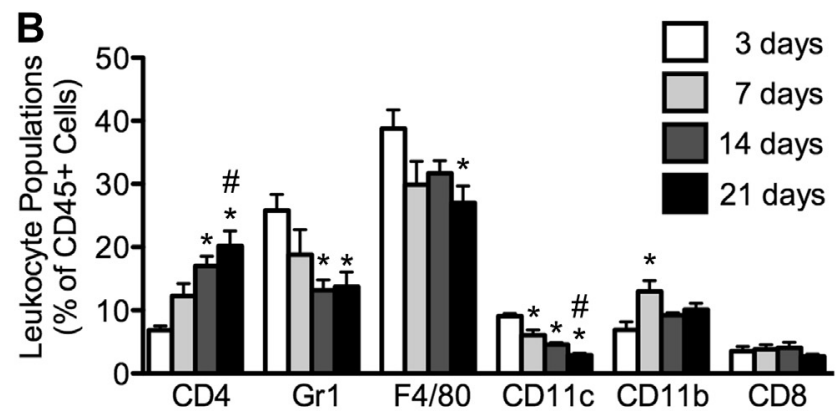

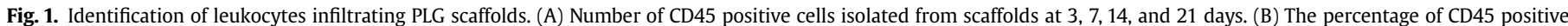

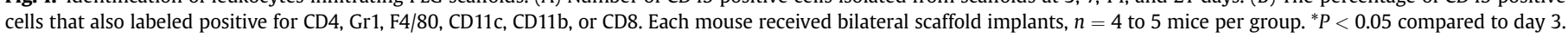
$\# P<0.05$ compared to day 7. Statistics determined by One Way ANOVA with Tukey post-hoc test.

\section{Results}

\subsection{Leukocyte infiltration into scaffolds}

Initial studies focused on characterizing dynamic leukocyte populations that infiltrate and reside within the microporous PLG scaffolds. Flow cytometry revealed that leukocytes, identified by CD45 expression, infiltrated the scaffold by day 3, and their numbers remained at similar levels through day 21 (Fig. 1A). However, the types of leukocytes associated with the scaffold changed during this time frame (Fig. 1B). Cells positive for F4/80, a macrophage marker, were the most numerous cell type at all time points, constituting $40 \%$ of the total CD 45 positive cells at day 3 but decreasing to $25 \%$ by day 21 . Similar trends were observed for cells positive for Gr1 and CD11c, which are markers for neutrophils and dendritic cells, respectively. In contrast, the proportion of CD4 positive cells significantly increased from $7 \%$ to $20 \%$ over this time frame, while CD8 positive cells consistently made up less than $5 \%$ of the cells in the scaffold. The remaining cells recovered from the scaffold were $\mathrm{CD} 11 \mathrm{~b}$ positive, but not positive for any other markers investigated. The presence of $\mathrm{CD} 11 \mathrm{~b}$ positive cells significantly increased from day 3 to day 7 and then returned to day 3 levels.

\subsection{Viral vector delivery from scaffolds}

We next investigated gene delivery as a means to locally induce protein production, which would be employed subsequently to express IL-10. Scaffolds loaded with luciferase lentivirus were implanted into the right IP fat pad. In vivo bioluminescence imaging indicated that luciferase expression within the IP fat region (Fig. 2A,
A

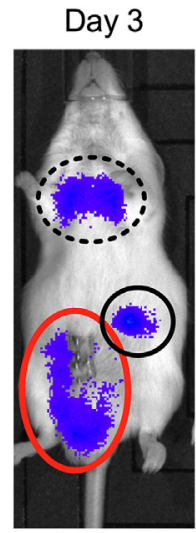

B

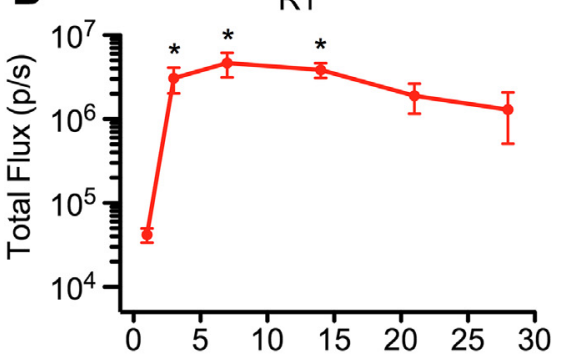

Day 7

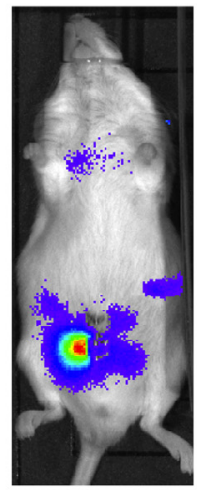

R1

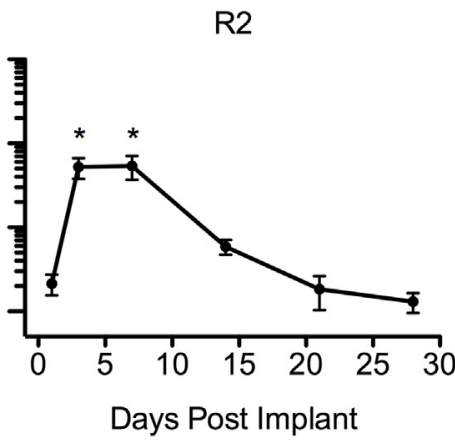

Day 28
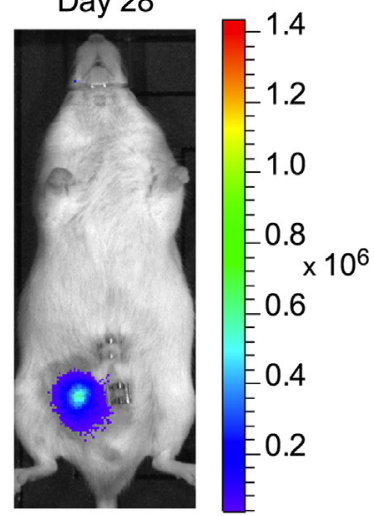

R2
R3

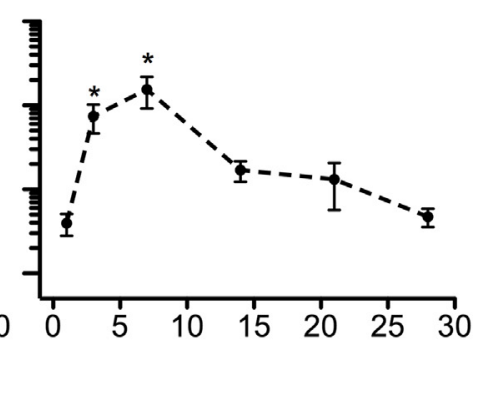

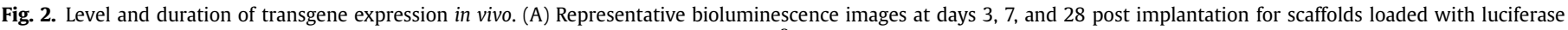

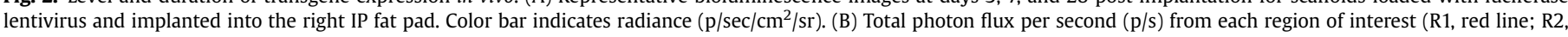

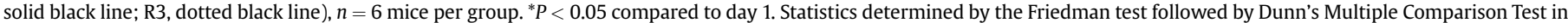

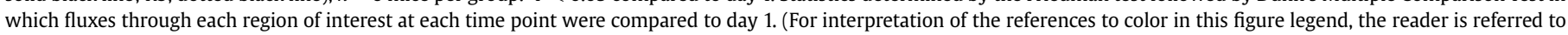
the web version of this article.) 

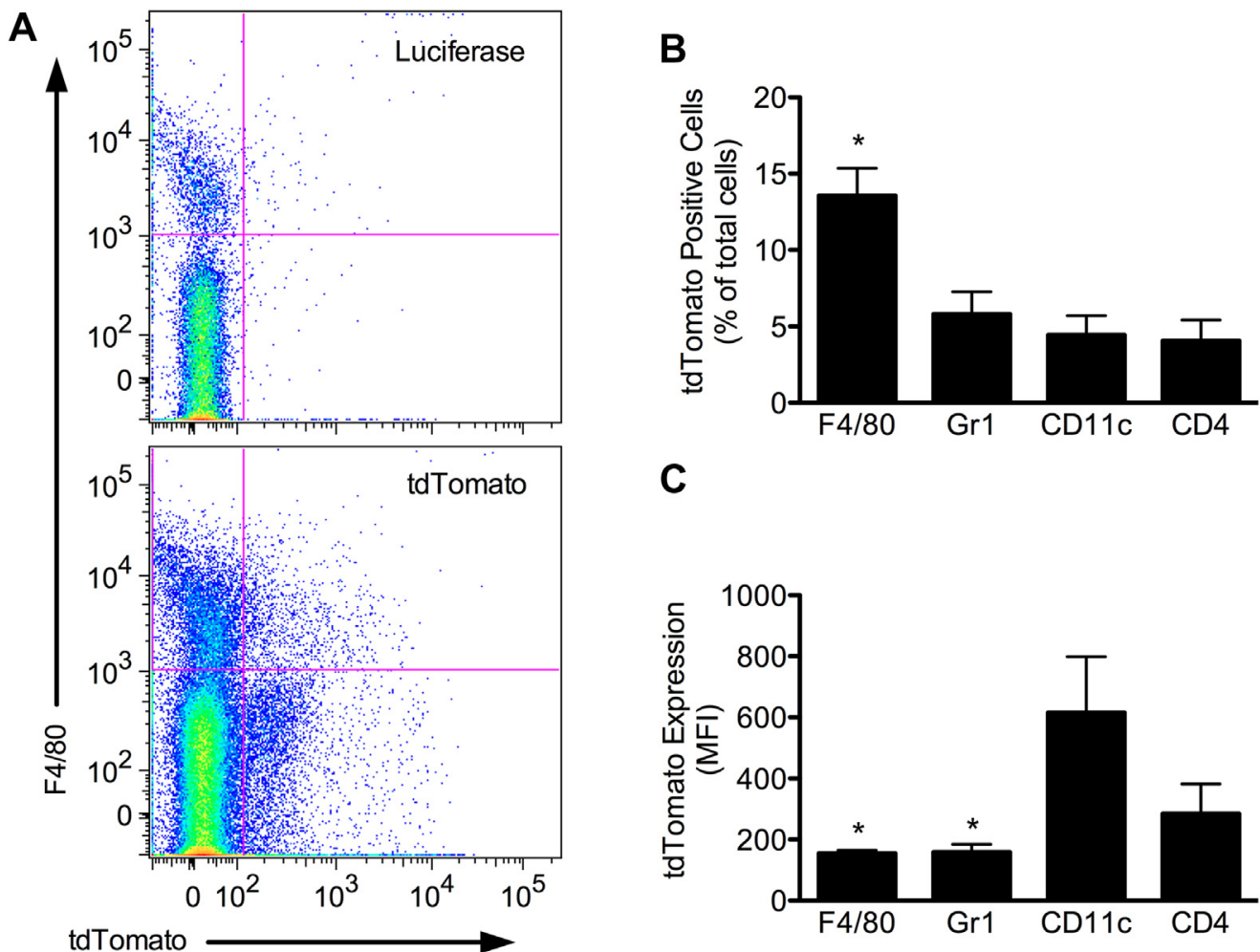

\section{C}

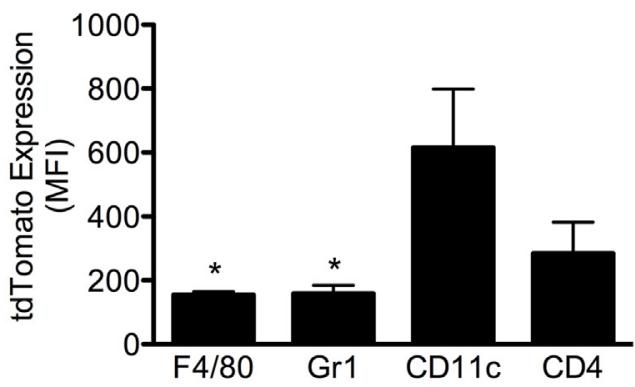

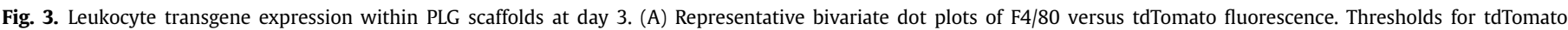

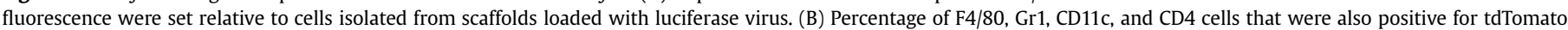

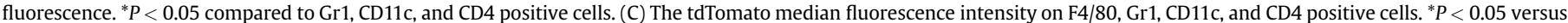
CD11c positive cells. Each mouse received bilateral scaffolds implants, $n=5$ mice per group. Statistics determined by one-way ANOVA with Tukey post-hoc test.

red line) was at background levels at day 1 , but maximal by day 3 and remained at similar levels through day 14 (Fig. 2B, R1). Luciferase expression in the IP fat region was heterogeneous within the group at days 21 and 28 and average levels were not significantly different from day 1. Luciferase expression was also observed in regions that correspond anatomically to the spleen (Fig. 2A, solid black line) and the thymus or lungs (Fig. 2A, dotted black line), which is consistent with previous reports and likely results from migration of leukocytes that were transduced at the scaffold to these tissues [19]. Luciferase expression at these sites was significantly above day 1 at days 3 and 7 (Fig. 2B, R2 and R3).

\subsection{Leukocyte transgene expression within scaffolds}

We subsequently investigated which leukocytes were transduced following lentivirus delivery. Transduced cells were
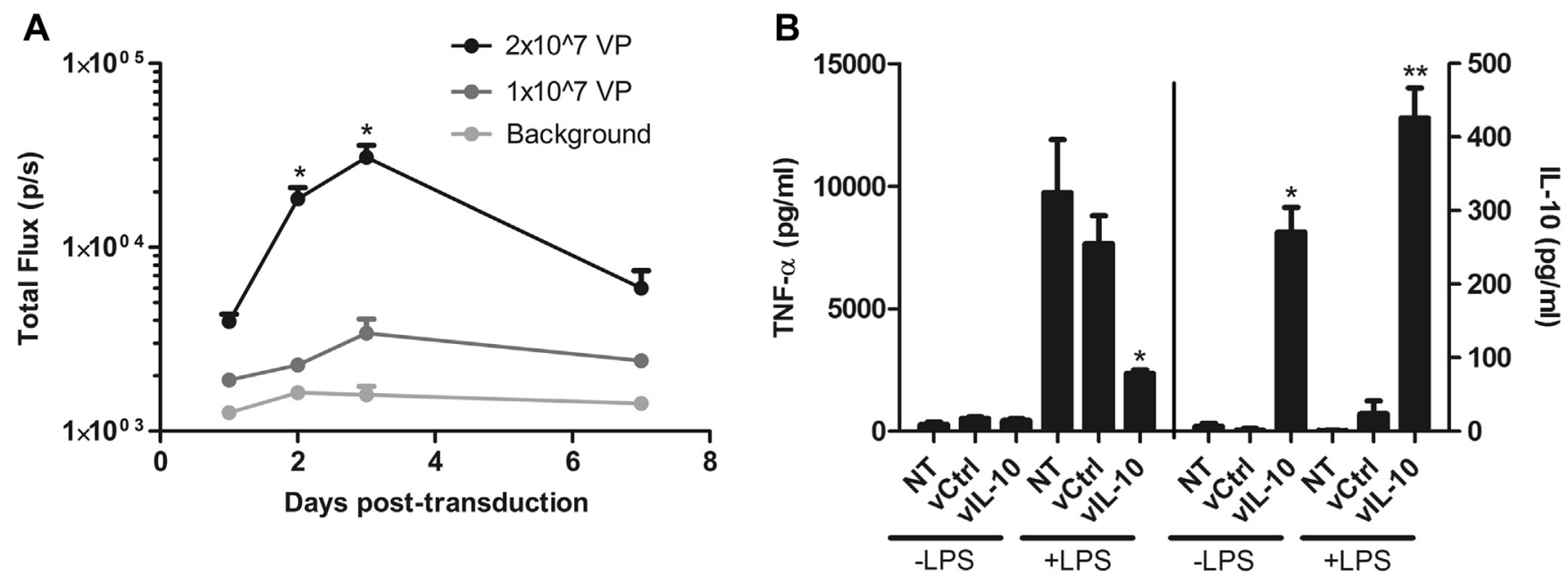

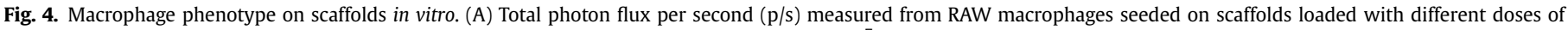

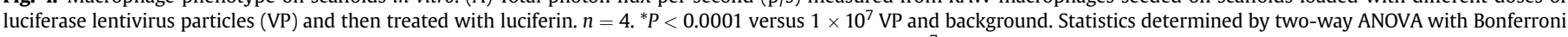

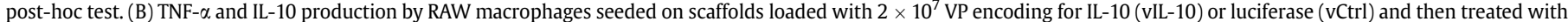

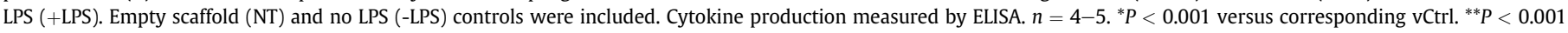
versus vIL-10 (-LPS). Statistics determined by one-way ANOVA with Tukey post-hoc test. 
determined by detection of tdTomato expression by flow cytometry, with the identity of these cells determined by antibody staining of surface markers (Fig. 3A). Three days after scaffold implant F4/80, Gr1, CD11c, and CD4 positive cells were positive for tdTomato expression. Approximately 15 percent of all $\mathrm{F} 4 / 80$ positive cells were positive for tdTomato expression, which was significantly greater than for Gr1, CD11c, and CD4 positive cells that ranged from $3 \%$ to $6 \%$ (Fig. 3B). In addition, approximately $13 \%$ of tdTomato positive cells were negative for leukocyte markers indicating other cell types such as adipocytes, fibroblasts, or endothelial cells were also transduced (data not shown). Interestingly, F4/80 and $\mathrm{Gr} 1$ positive cells had the lowest mean fluorescence intensity for tdTomato, suggesting relatively low levels of protein production per cell or high levels of protein degradation (Fig. 3C). In contrast, CD11c positive cells had the greatest fluorescence intensity for tdTomato per cell.

\subsection{Macrophage phenotype on scaffolds in vitro}

Gene delivery from PLG scaffolds was subsequently investigated in vitro to determine if macrophage phenotype could be modulated, as measured by a reduction in TNF- $\alpha$ production and an increase in IL-10 production. A lentivirus dose of $2 \times 10^{7}$ viral particles (VP) was determined to be necessary for achieving transgene expression levels that were significantly above background (Fig. 4A). Gene expression by the cells was observed to increase through day 3 , and subsequently decline through day 7. IL-10 lentivirus delivery from the scaffold, followed by LPS-induction of cytokine production at day 3 (peak transgene expression), resulted in a significant decrease in TNF- $\alpha$ production relative to no treatment and virus control (Fig 4B). Furthermore, a significant increase in IL-10 production was observed relative to control virus, no treatment, and LPS negative controls. These data indicate the scaffold based viral delivery of IL-10 is capable of modulating macrophage cytokine expression in response to an acute inflammatory stimulus.

\subsection{Leukocyte infiltration into IL-10 virus releasing scaffolds}

We hypothesized that localized IL-10 expression from the scaffold would modulate the infiltration of leukocytes and their phenotype. At 3 days post implantation of IL-10 lentivirus loaded scaffolds, the number of leukocytes in the scaffold was not significantly different between the scaffolds loaded with an IL-10 or luciferase control virus. However, seven days post implantation, leukocyte infiltration significantly decreased relative to control (Fig. 5A). At both time points, the scaffolds loaded with luciferase virus had similar numbers of leukocytes compared to blank scaffolds (Fig. 1A), indicating that vector delivery did not significantly affect leukocyte infiltration. Both the number of F4/80 and Gr1 positive cells was significantly decreased on day 7 within scaffolds loaded with IL-10 virus compared to scaffolds loaded with luciferase virus (Fig. 5B). However, at day 7, the relative percentages of leukocyte populations were not significantly changed for the cell types investigated, though the greatest trend was a decrease in the percentage of $\mathrm{F} 4 / 80$ positive cells (Fig. $5 \mathrm{C}$ ).

\subsection{Cytokine production within IL-10 virus releasing scaffolds}

A hallmark of the host response is inflammatory cytokine expression such as IL- $1 \beta$, IFN- $\gamma$, and TNF- $\alpha$, while resolution of inflammation is characterized by IL-10 expression. Flow cytometry indicated that $\mathrm{F} 4 / 80$ positive cells isolated from IL-10 releasing scaffolds had a greater mean fluorescence intensity following IL-10 antibody staining relative to $\mathrm{F} 4 / 80$ positive cells isolated from luciferase releasing scaffolds, indicating elevated IL-10 expression
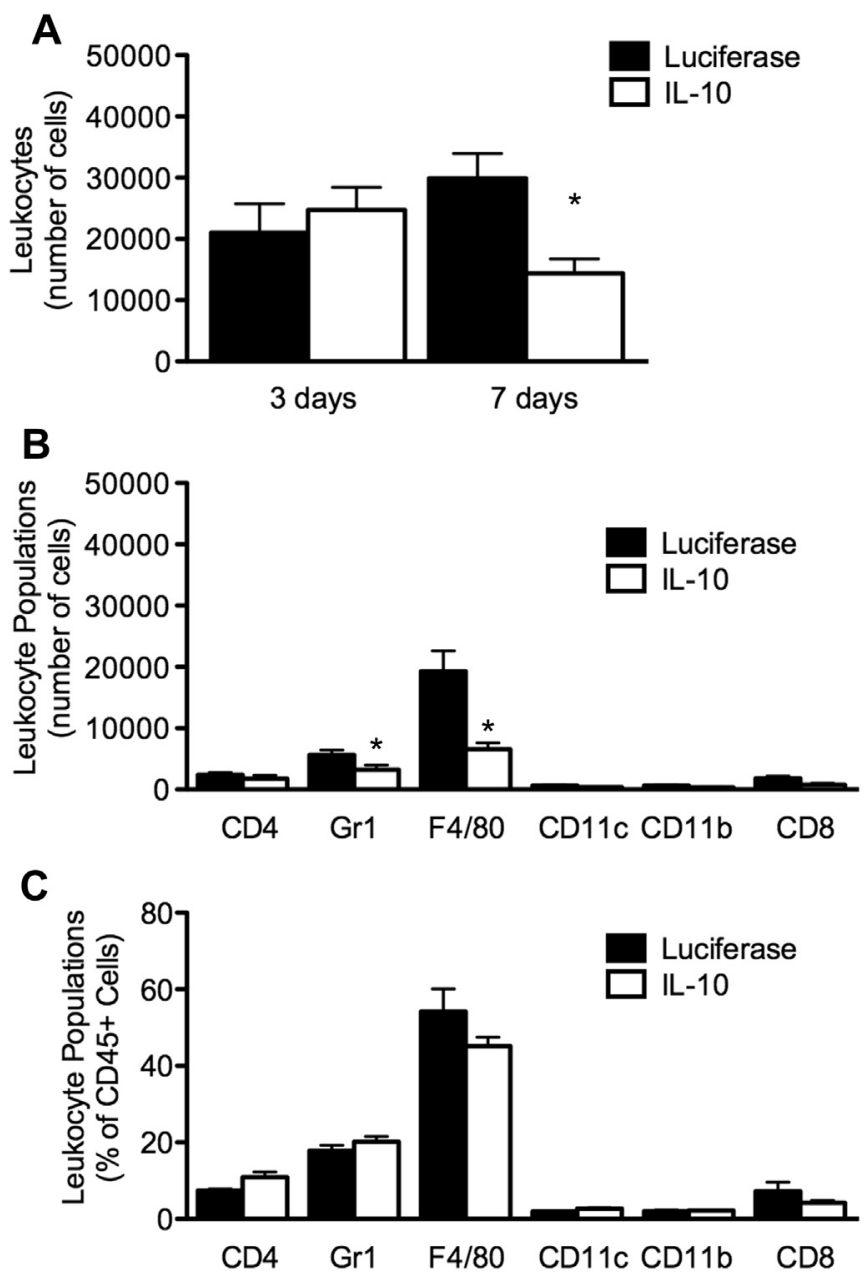

Fig. 5. Leukocyte infiltration into IL-10 virus releasing scaffolds. (A) Number of CD45 positive cells isolated from scaffolds loaded with IL-10 or luciferase virus at day 3 and day 7 post implantation. ${ }^{*} P<0.05$ versus day 7 luciferase. (B) Number of CD4, Gr1, F4/ 80, CD11c, CD11b, or CD8 cells isolated from scaffolds loaded with IL-10 or luciferase virus at day 7. (C) The percentage of CD45 positive cells that were CD4, Gr1, F4/80 CD11c, CD11b, or CD8 positive at day $7 .{ }^{*} P<0.05$ versus luciferase for each cell type. Each mouse received bilateral scaffolds implants. 5 mice per group. Statistics determined by $t$-test.

(Fig. 6A). Furthermore, in IL-10 releasing scaffolds, F4/80 positive cells expressed significantly more IL-10 than did CD4 and CD11c positive cells. No significant difference in IL-10 expression in CD4 and $\mathrm{CD} 11 \mathrm{c}$ positive cells was detected between the two groups. We next investigated expression of the pro-inflammatory cytokines IL$1 \beta$, IFN- $\gamma$, and TNF- $\alpha$ (Fig. 6B-D). In scaffolds delivering IL-10, we observed that CD4, F4/80, and CD11c positive cells expressed significantly less IFN- $\gamma$ compared to cells isolated from control scaffolds. No significant differences in intracellular TNF- $\alpha$ and IL-1 $\beta$ expression were observed between the two groups; however, TNF$\alpha$ exhibited a decreasing trend for all cell types.

\section{Discussion}

The innate immune response to the implanted biomaterials contributes to the post-injury microenvironment that influences the extent of regeneration [1,2]. Excessive inflammation can impede the implant's integration with the host and tissue regeneration, and can adversely affect the survival and function of cells delivered by the scaffold [1]. We characterized the host response to porous PLG scaffolds implanted in the IP fat, an anatomical site that 
A

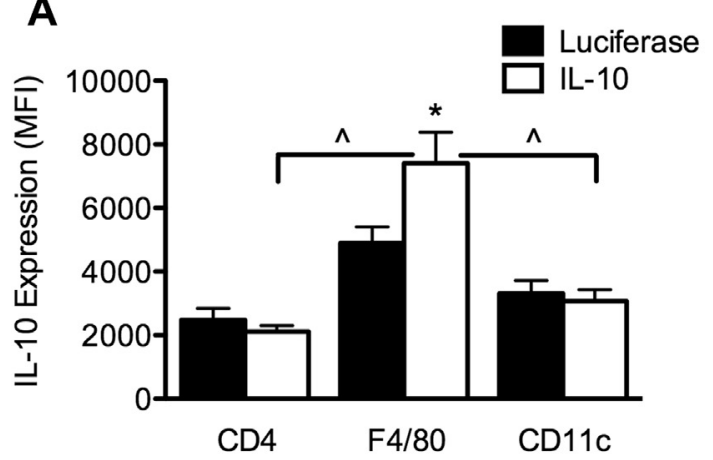

C

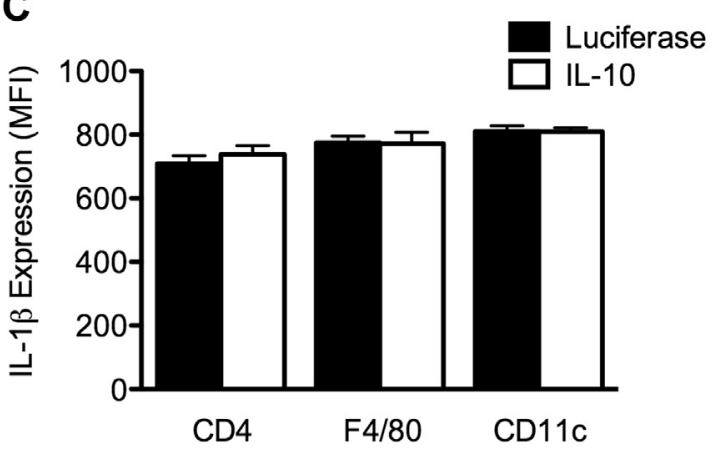

B

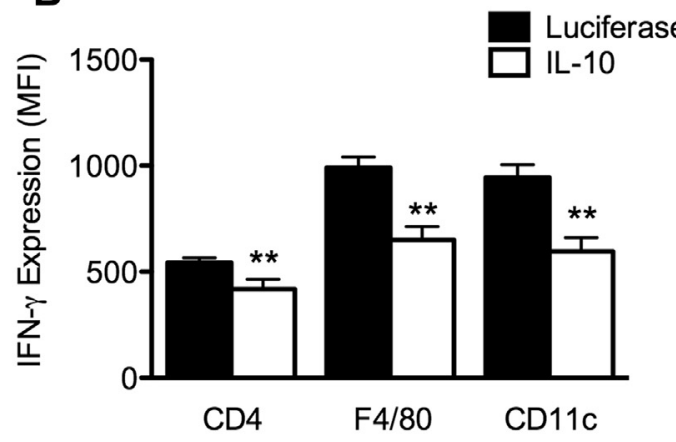

D

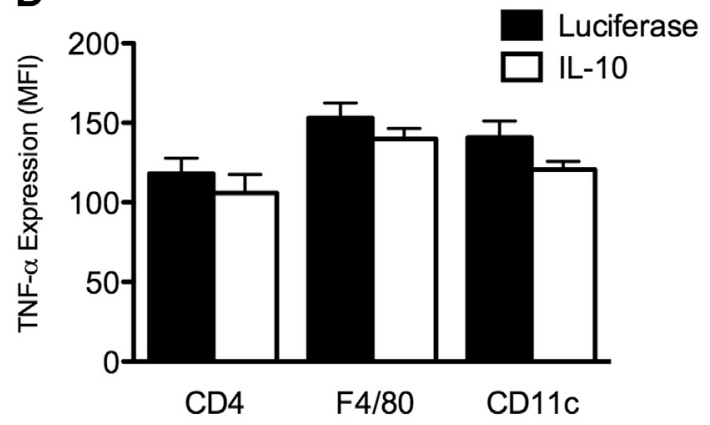

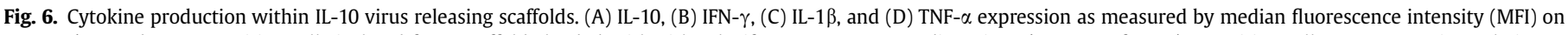

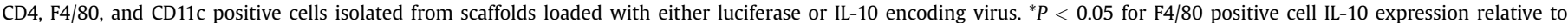

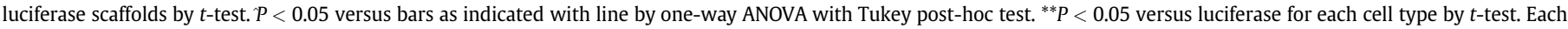
mouse received bilateral scaffolds implants, $n=5$ mice per group.

has been employed for cell transplantation, such as islets for diabetes therapies [25-29]. Multicolor flow cytometry was used to characterize the kinetics of leukocyte infiltration of six major leukocyte populations within the scaffold at 4 time points postimplant. Leukocytes were present within the scaffold as early as day 3 and remained at similar numbers through day 21. Macrophages were the most prevalent leukocyte found in the scaffold at all time points, consistent with previous studies [11,18,20,21,24,30]. The studies herein also quantified the frequency of other leukocyte populations, and detected members of the adaptive immune system such as T cells. Thus strategies to modulate inflammation, and ultimately immunity, within biomaterial implants will need to influence cellular processes (e.g., leukocyte infiltration) at or near the time of implantation.

IL-10 delivery decreased leukocyte numbers within the scaffold, an effect observed at day 7 but not day 3 . The inability to influence leukocyte numbers at day 3 likely results from the gene expression profile of the IL-10, which in vivo bioluminescence imaging suggests was low prior to day 3 and peaks between day 3 and 14. The decreased leukocyte numbers within the scaffold was primarily due to a decrease in the number of macrophages. The ability to decrease macrophage infiltration is significant in the context of tissue engineering as their adhesion to biomaterial scaffolds can result in macrophage activation [31,32]. Macrophage activation involves the release of cytokines, which recruit and activate additional leukocytes leading to impairment of wound healing [8], clinical failure of medical devices [33], and the rejection of nonautologous tissue. The significance of reduced macrophage infiltration is exemplified with studies of islet transplantation. Macrophage infiltration into islet grafts results in early rejection, while systemic macrophage depletion significantly improves islet graft survival [34-36]. A consequence of systemic macrophage depletion would be an impaired host defense and wound healing $[37,38]$.
Thus, a localized strategy to exclude macrophages from the biomaterial or transplant site could create a microenvironment more favorable to survival of transplanted cells while minimizing the risk of systemic consequences.

A cellular level analysis, enabled by flow cytometry, indicated that following viral IL-10 delivery from scaffolds, macrophages upregulated IL-10, while macrophages, dendritic cells, and CD4 T cells down-regulated the production of the inflammatory cytokine IFN$\gamma$. Many studies have characterized bulk cytokine expression within and around implanted biomaterial scaffolds [6,7,11]. Herein, we identify the cytokines levels within specific cell types in situ. Macrophages were the highest producers of IL-10 following IL-10 gene delivery, which likely contributed to the reduction in leukocyte infiltration into the scaffold. Decreased IFN $-\gamma$ levels within macrophages, dendritic cells, and $\mathrm{T}$ cells were observed within scaffolds inducing IL-10 expression. IFN- $\gamma$ typically converts resting macrophages into cells with increased capacity for antigen presentation, pro-inflammatory cytokine synthesis, and phagocytosis [39], thus playing a key role in allogeneic tissue rejection [40]. The ability to modulate both IL-10 and IFN- $\gamma$ production within the scaffold would be expected to contribute to a more favorable environment for regeneration or survival and function of transplanted cells.

Leukocytes were transduced directly with delivery of the gene therapy vector, with macrophages being the most transduced leukocyte and dendritic cells exhibiting the greatest intracellular transgene-encoded protein production. Previous studies have reported that both macrophages and dendritic cells exhibit transgene expression within plasmid [18,24,30] and viral-based [19] gene releasing scaffolds. Histological analysis identified these cells within the scaffold and preferentially located near the polymer surface, yet histology was unable to identify transgene expression levels. Despite producing less tdTomato than dendritic cells, 
macrophages were the greatest producer of IL-10 following delivery of the lentivirus encoding IL-10. This discrepancy in protein production could be due to positive feedback loops present in macrophages, in which IL-10 signaling induces increased IL-10 production [41]. Viral delivery is also known to transduce nonleukocytes present in and around the scaffold [19,20,42] and our results here indicate that a subset of tdTomato positive cells were not leukocytes. Thus adipocytes, fibroblasts, or endothelial cells present around the scaffolds likely also contributed to IL-10 transgene expression responsible for the leukocyte numbers and cytokine expression observed within the scaffold.

\section{Conclusions}

Following scaffold implantation into the intraperitoneal fat, leukocyte infiltration reached maximal levels by day 3-post implant and remained at similar levels through day 21, with macrophages being the most prevalent cell type within the scaffold at all time points. Delivery of an IL-10 encoding vector significantly decreased leukocyte infiltration, increased macrophage IL-10 expression, and decreased macrophage, dendritic cell, and CD4 T cell IFN- $\gamma$ expression. Macrophages were the most commonly transduced leukocyte in this system, while transduced dendritic cells expressed the highest level of the transgene, indicating that modulation of leukocyte infiltration and IFN- $\gamma$ expression likely occurred through both autocrine and paracrine signaling mechanisms. This study characterized the role that leukocyte populations play in modulating the local immune environment following IL-10 expression at tissue engineering scaffolds, which has implications for the design of combinatorial delivery devices for local immunomodulation.

\section{Acknowledgments}

Financial support for this research was provided by the National Institutes of Biomedical Imaging and Bioengineering (NIBIB) at the National Institutes of Health (NIH) through grant number R01 EB009910, R01 EB005678, and R01 CA173745. This work was also supported by the Northwestern University RHLCCC Flow Cytometry Facility and a Cancer Center Support Grant (NCI CA060553).

\section{Appendix A. Supplementary data}

Supplementary data related to this article can be found at http:// dx.doi.org/10.1016/j.biomaterials.2013.11.036.

\section{References}

[1] Anderson JM, Rodriguez A, Chang DT. Foreign body reaction to biomaterials. Semin Immunol 2008;20:86-100.

[2] Jones KS. Effects of biomaterial-induced inflammation on fibrosis and rejection. Semin Immunol 2008;20:130-6.

[3] Donnelly DJ, Popovich PG. Inflammation and its role in neuroprotection, axonal regeneration and functional recovery after spinal cord injury. Exp Neurol 2008;209:378-88.

[4] Lopez-Armada MJ, Sanchez-Pernaute O, Largo R, Diez-Ortego I, Palacios I, Egido J, et al. Modulation of cell recruitment by anti-inflammatory agents in antigen-induced arthritis. Ann Rheum Dis 2002;61:1027-30.

[5] Gonzalez R, Glaser J, Liu MT, Lane TE, Keirstead HS. Reducing inflammation decreases secondary degeneration and functional deficit after spinal cord injury. Exp Neurol 2003;184:456-63.

[6] Brodbeck WG, Voskerician G, Ziats NP, Nakayama Y, Matsuda T, Anderson JM. In vivo leukocyte cytokine mRNA responses to biomaterials are dependent on surface chemistry. J Biomed Mater Res A 2003;64:320-9.

[7] Gretzer C, Emanuelsson L, Liljensten E, Thomsen P. The inflammatory cell influx and cytokines changes during transition from acute inflammation to fibrous repair around implanted materials. J Biomater Sci Polym Ed 2006;17: $669-87$.

[8] Broughton 2nd G, Janis JE, Attinger CE. The basic science of wound healing. Plast Reconstr Surg 2006;117:12S-34S.
[9] Wood KJ, Goto R. Mechanisms of rejection: current perspectives. Transplantation 2012:93:1-10.

[10] Moore KW, de Waal Malefyt R, Coffman RL, O'Garra A. Interleukin-10 and the interleukin-10 receptor. Annu Rev Immunol 2001;19:683-765.

[11] Holladay C, Power K, Sefton M, O'Brien T, Gallagher WM, Pandit A. Functionalized scaffold-mediated interleukin 10 gene delivery significantly improves survival rates of stem cells in vivo. Mol Ther 2011;19:969-78.

[12] Hong YS, Laks H, Cui G, Chong T, Sen L. Localized immunosuppression in the cardiac allograft induced by a new liposome-mediated IL-10 gene therapy. J Heart Lung Transplant 2002;21:1188-200.

[13] Dudler J, Li J, Pagnotta M, Pascual M, von Segesser LK, Vassalli G. Gene transfer of programmed death ligand-1.Ig prolongs cardiac allograft survival. Transplantation 2006:82:1733-7.

[14] Montane J, Bischoff L, Soukhatcheva G, Dai DL, Hardenberg G, Levings MK, et al. Prevention of murine autoimmune diabetes by CCL22-mediated Treg recruitment to the pancreatic islets. J Clin Invest 2011;121:3024-8.

[15] Richardson TP, Peters MC, Ennett AB, Mooney DJ. Polymeric system for dua growth factor delivery. Nat Biotechnol 2001;19:1029-34.

[16] Thevenot PT, Nair AM, Shen J, Lotfi P, Ko CY, Tang L. The effect of incorporation of SDF-1alpha into PLGA scaffolds on stem cell recruitment and the inflammatory response. Biomaterials 2010;31:3997-4008.

[17] Jang JH, Houchin TL, Shea LD. Gene delivery from polymer scaffolds for tissue engineering. Expert Rev Med Devices 2004;1:127-38.

[18] Aviles MO, Lin CH, Zelivyanskaya M, Graham JG, Boehler RM, Messersmith PB et al. The contribution of plasmid design and release to in vivo gene expression following delivery from cationic polymer modified scaffolds. Biomaterials 2010:31:1140-7.

[19] Aviles MO, Shea LD. Hydrogels to modulate lentivirus delivery in vivo from microporous tissue engineering scaffolds. Drug Deliv Transl Res 2011;1:91101.

[20] Boehler RM, Shin S, Fast AG, Gower RM, Shea LD. A PLG/HAp composite scaffold for lentivirus delivery. Biomaterials 2013;34:5431-8.

[21] Holladay CA, Duffy AM, Chen X, Sefton MV, O'Brien TD, Pandit AS. Recovery of cardiac function mediated by MSC and interleukin-10 plasmid functionalised scaffold. Biomaterials 2012;33:1303-14.

[22] Miyoshi H, Blomer U, Takahashi M, Gage FH, Verma IM. Development of a self inactivating lentivirus vector. J Virol 1998;72:8150-7.

[23] Jang JH, Rives CB, Shea LD. Plasmid delivery in vivo from porous tissueengineering scaffolds: transgene expression and cellular transfection. Mo Ther 2005; $12: 475-83$.

[24] Rives CB, des Rieux A, Zelivyanskaya M, Stock SR, Lowe Jr WL, Shea LD. Layered PLG scaffolds for in vivo plasmid delivery. Biomaterials 2009;30:394-401.

[25] Blomeier H, Zhang X, Rives C, Brissova M, Hughes E, Baker M, et al. Polymer scaffolds as synthetic microenvironments for extrahepatic islet transplantation. Transplantation 2006;82:452-9.

[26] Graham JG, Zhang X, Goodman A, Pothoven K, Houlihan J, Wang S, et al. PLC scaffold delivered antigen-specific regulatory $\mathrm{T}$ cells induce systemic tolerance in autoimmune diabetes. Tissue Eng Part A 2013;19:1465-75.

[27] Gibly RF, Zhang X, Graham ML, Hering BJ, Kaufman DB, Lowe Jr WL, et al Extrahepatic islet transplantation with microporous polymer scaffolds in syngeneic mouse and allogeneic porcine models. Biomaterials 2011:32:967784.

[28] Salvay DM, Rives CB, Zhang X, Chen F, Kaufman DB, Lowe Jr WL, et al Extracellular matrix protein-coated scaffolds promote the reversal of diabetes after extrahepatic islet transplantation. Transplantation 2008;85:1456-64.

[29] van der Windt DJ, Echeverri GJ, Ijzermans JN, Cooper DK. The choice of anatomical site for islet transplantation. Cell Transplant 2008;17:1005-14.

[30] Salvay DM, Zelivyanskaya M, Shea LD. Gene delivery by surface immobilization of plasmid to tissue-engineering scaffolds. Gene Ther 2010;17:1134-41.

[31] McNally AK, Jones JA, Macewan SR, Colton E, Anderson JM. Vitronectin is a critical protein adhesion substrate for IL-4-induced foreign body giant cell formation. J Biomed Mater Res A 2008;86:535-43.

[32] Jenney CR, Anderson JM. Adsorbed serum proteins responsible for surface dependent human macrophage behavior. J Biomed Mater Res 2000;49:43547.

[33] Wisniewski N, Moussy F, Reichert WM. Characterization of implantable biosensor membrane biofouling. Fresenius J Anal Chem 2000;366:611-21.

[34] Rossi L, Migliavacca B, Pierige F, Serafini S, Sanvito F, Olivieri S, et al. Prolonged islet allograft survival in diabetic mice upon macrophage depletion by clodronate-loaded erythrocytes. Transplantation 2008;85:648-50.

[35] Kaufman DB, Platt JL, Rabe FL, Dunn DL, Bach FH, Sutherland DE. Differentia roles of Mac- $1+$ cells, and $\mathrm{CD} 4+$ and $\mathrm{CD} 8+\mathrm{T}$ lymphocytes in primary nonfunction and classic rejection of islet allografts. J Exp Med 1990:172:291-302.

[36] Bottino R, Fernandez LA, Ricordi C, Lehmann R, Tsan MF, Oliver R, et al. Transplantation of allogeneic islets of Langerhans in the rat liver: effects of macrophage depletion on graft survival and microenvironment activation. Diabetes 1998;47:316-23.

[37] Li S, Li B, Jiang H, Wang Y, Qu M, Duan H, et al. Macrophage depletion impairs corneal wound healing after autologous transplantation in mice. PLoS One 2013:8:e61799.

[38] van Amerongen MJ, Harmsen MC, van Rooijen N, Petersen AH, van Luyn MJ Macrophage depletion impairs wound healing and increases left ventricula remodeling after myocardial injury in mice. Am J Pathol 2007;170:818-29.

[39] Martinez FO, Helming L, Gordon S. Alternative activation of macrophages: an immunologic functional perspective. Annu Rev Immunol 2009;27:451-83. 
[40] Hidalgo LG, Halloran PF. Role of IFN-gamma in allograft rejection. Crit Rev Immunol 2002:22:317-49.

[41] Staples KJ, Smallie T, Williams LM, Foey A, Burke B, Foxwell BM, et al. IL-10 induces IL-10 in primary human monocyte-derived macrophages via the transcription factor Stat3. J Immunol 2007;178:4779-85.
[42] Tuinstra HM, Aviles MO, Shin S, Holland SJ, Zelivyanskaya ML, Fast AG, et al, Multifunctional, multichannel bridges that deliver neurotrophin encoding lentivirus for regeneration following spinal cord injury. Biomaterials 2012;33: $1618-26$. 